\title{
Inserção no mercado de trabalho e uso de drogas entre escolares de duas cidades de médio porte do sul do Brasil
}

\author{
Labour market and drug use among schoolchildren \\ of two medium size cities in southern Brazil
}

Simone Poletto', Rogério Lessa Horta', Vanessa Andina Teixeira', Valenca Lemes Grapiglia', Alexandre Dido Balbinot ${ }^{\top}$

\section{RESUMO}

Objetivo: $O$ início do consumo de drogas ocorre, geralmente, na adolescência. Entre os fatores associados a esse comportamento, está o trabalho. Este artigo analisa a associação entre a inserção no mercado de trabalho e o uso no ano de álcool, tabaco, maconha e cocaína, em uma amostra de 1.961 escolares de dois municípios de médio porte do Rio Grande do Sul. Métodos: Foram estimadas razões de prevalência brutas e ajustadas, primeiramente, para sexo e idade e, posteriormente, para sexo, idade e supervisão do uso de internet no domicílio. Resultados: Na análise bruta, a associação com a inserção no mercado de trabalho foi significativa para uso no ano de álcool, tabaco, maconha e cocaína. Na estimativa de razões de prevalência ajustadas para sexo e idade, apenas a associação com uso no ano de tabaco se manteve e desapareceu ao ajustar também para a variável de supervisão parental do uso da internet. Conclusões: $\bigcirc$ fato de a associação não se manter após a análise ajustada indica que, entre escolares, portanto, adolescentes ainda vinculados à escola, inserção no mercado de trabalho e uso no ano daquelas substâncias se expandem de modo semelhante, mas muito mais influenciados pelo avanço da idade, por especificidades de gênero e de acordo com modelos de cuidado no ambiente doméstico.

\begin{abstract}
Objective: Having a paid work appears among the factors associated with the onset of drug usage by teenagers. This paper aims to estimate the association between the insertion in the labor market and last year usage of alcohol, tobacco, marijuana or cocaine in a sample of 1,961 students aging 14 to 17 on two medium-sized cities in southern Brazil. Methods: Prevalence ratios were estimated, first adjusted for sex and age, and then adjusted also for sex, age, and then to sex, age, and supervision of internet use at home. Results: Prevalence rates indicated significant association among the insertion in the labor market and the consumption of the interest substances, but when adjusting for gender and age, only the association with tobacco use remained significant and it disappeared when parent's supervision was included in the model of analysis. Conclusions: Data show that among adolescents yet linked
\end{abstract}

1 Universidade do Vale do Rio dos Sinos (Unisinos), Programa de Pós-Graduação em Saúde Coletiva.

$$
\begin{gathered}
\text { Recebido em } \\
\text { 24/2/2015 } \\
\text { Aprovado em } \\
\text { 17/4/2015 }
\end{gathered}
$$

DOI: $10.1590 / 0047-2085000000069$
Endereço para correspondência: Rogério Lessa Horta

Rua Jari, 671, ap. 703, Passo D’Areia

91350-170 - Porto Alegre, RS, Brasil

E-mail: rogeriohortamed@gmail.com 


\section{Keywords}

Alcohol, tobacco, street

drugs, work, student,

teenager. to school, enter the job market and use of those substances in the year expand similarly, but much more influenced by advancing age, according to gender specificities and according to patterns of care at home.

\section{INTRODUÇÃO}

A adolescência é um período no qual o jovem desenvolve maior autonomia em relação a seus cuidadores e afirma sua identidade e individualidade, além de estreitar vínculos com seus pares ${ }^{1,2}$. Esse período é marcado por diversas mudanças de ordem biológica, psicológica e social, tornando o adolescente mais suscetível às influências do meio ${ }^{3,4}$. Essa condição suscita grande número de experimentações e descobertas e os mercados de álcool, tabaco e outras drogas estão atentos a esse público ${ }^{5}$.

Em 2010, considerando a população combinada das 27 capitais de estados brasileiros, foram estimadas as seguintes prevalências de uso no ano, entre escolares: álcool - 42,4\%, tabaco $-9,6 \%$, maconha - 3,7\%, e cocaína ou crack $-2,3 \%{ }^{6}$. O uso de drogas na adolescência pode ter diferentes impactos, desde problemas de saúde até o ajustamento social, além de queda no rendimento escolar e prejuízo no desenvolvimento das habilidades cognitivas, comportamentais e emocionais $^{7,8}$. A preocupação com o fenômeno não se restringe apenas aos riscos inerentes ao comportamento, mas ao fato de estar sendo detectado de modo mais precoce nas últimas décadas ${ }^{7}$. No Brasil, a propaganda de bebidas alcoólicas é direcionada principalmente a adolescentes e adultos jovens e a exposição a esse tipo de marketing amplia ou favorece o consumo, além de antecipar o início do uso, principalmente entre pré-adolescentes ${ }^{5}$.

Diversos fatores são apontados como associados ao uso de drogas na adolescência, entre eles: problemas no relacionamento com os pais ${ }^{8-10}$, estilos parentais superprotetores ou liberais ${ }^{8,9}$, presença em casa de familiar usuário de drogas $^{2,9-11}$, sentir-se pouco compreendido na família ${ }^{4}$, além de vivência de maus-tratos ${ }^{9}$, ter sido assaltado ou roubado no ano anterior ${ }^{9}$ e ausência de prática religiosa ${ }^{4,9}$. A relação entre os pares também tem mostrado associação com o uso de drogas entre adolescentes, particularmente quando eles têm comportamentos de tolerância e aprovação ou se consomem drogas ${ }^{8}$. Variáveis relacionadas à vida escolar também são citadas, entre elas, estudar no período noturno ${ }^{4} \mathrm{e}$ ter baixo rendimento escolar 8 ,

A inserção no mercado de trabalho vem sendo investigada como um possível fator associado ao uso de substâncias psicoativas entre adolescentes ${ }^{4,9,11-15}$. Estudo realizado na Irlanda do Norte ${ }^{12}$ apontou que jovens que tinham empregos de meio período apresentaram maior probabilidade de uso de drogas lícitas. Em Pelotas (RS) ${ }^{10}$, um estudo longitudinal apontou que, entre os jovens inseridos no mercado de tra- balho, a probabilidade de fumar tabaco era $75 \%$ maior. Outros estudos, realizados na Coreia do Sul13 ${ }^{3}$, Campinas (SP) ${ }^{4}$, Midwest (EUA) ${ }^{15}$ e Baltimore (EUA) $)^{14}$ e estudo de revisão de documentos do NHSDA (The National Household Survey on Drug Abuse) ${ }^{16}$, encontraram associação significativa entre ter um trabalho e crescimento nas prevalências de uso de drogas. Além da relação direta entre trabalho e uso de substâncias, a inserção de adolescentes em atividades profissionais também tem sido descrita como associada a outras variáveis que também influenciam os comportamentos de consumo de álcool e outras drogas. Entre elas, o trabalho parece meIhorar autoestima, desenvolvimento de responsabilidades e autonomia $^{16,17}$, mas também baixo rendimento escolar ${ }^{15,17}$, ferimentos relacionados ao trabalho ${ }^{16}$, agravos frequentes à saúde e prejuízos sociais ${ }^{17}$.

No Brasil, a preocupação com os riscos inerentes à exposição aos ambientes e condições de trabalho levou a uma regulamentação, que estabelece a inserção de adolescentes no mercado de trabalho apenas a partir de 14 anos de idade, lei regulamentada em 2000 como a Lei do Menor Aprendiz. Segundo a norma, a partir dessa idade, jovens podem ser empregados apenas na condição de aprendizes, desde que o emprego não acarrete danos físicos, morais, sociais ou psicológicos e que, mesmo trabalhando, ele possa continuar frequentando a escola ${ }^{18}$.

Este artigo avalia a relação entre prática de atividade remunerada e uso de substâncias psicoativas, entre jovens com idades entre 14 e 17 anos de idade, em municípios de médio porte do sul do Brasil, não contemplados nos estudos revisados.

\section{MÉTODOS}

Estudo transversal, de base escolar, realizado em 2012 com uma amostra representativa dos estudantes de todas as escolas, tanto nas redes públicas (estadual e municipal) quanto na rede privada, das cidades de Lajeado e Sapiranga (RS). Trata-se de dois municípios de porte médio, do interior do estado do Rio Grande do Sul, perfil usualmente não contemplado nos grandes estudos nacionais. Lajeado é localizada na região noroeste do estado, situando-se a $117 \mathrm{~km}$ da capital, Porto Alegre. Sapiranga é localizada na região do Vale do Rio dos Sinos, situando-se a 60 km de distância da capital. Os municípios tinham, à época da coleta de dados, cerca de 70 mil habitantes, cada um. O município de Lajeado tinha aproximadamente 15 mil matrículas distribuídas entre as redes escolares 
existentes, sendo cerca de 6,5 mil relativas a jovens na faixa de 12 a 17 anos, distribuídos entre escolas da rede pública municipal, estadual e privada. O município de Sapiranga tinha na sua rede educacional 15,8 mil matrículas, distribuídas entre as redes escolares, sendo aproximadamente 8,9 mil relativas a jovens da faixa etária de interesse. No município de Lajeado, os escolares se distribuíam em 33 escolas e, em Sapiranga, em 22, totalizando 55 escolas incluídas. Todas foram visitadas.

A amostra foi composta por 3.547 estudantes de 12 a 17 anos, matriculados no ensino fundamental (a partir do 70 ano) e no ensino médio. Os dados foram coletados por meio de questionários autoaplicados e anônimos. Foi realizada amostragem aleatória por turma, preservando a proporcionalidade da amostra por sexo, idade e rede de ensino (pública municipal, pública estadual e privada). Todas as turmas de todas as escolas foram relacionadas para sorteio. Ao todo, foram visitadas 214 turmas nas escolas de Lajeado (RS) e 75 turmas nas escolas de Sapiranga (RS).

Para este estudo, foram selecionados os estudantes com 14 anos de idade ou mais, considerando-se que nessa faixa etária a inserção no mercado de trabalho se torna legal|18. A subamostra para esta análise foi composta por 1.999 estudantes, apenas aqueles com idades entre 14 e 17 anos, matriculados entre 070 ano do ensino fundamental e o $3^{\circ}$ ano do ensino médio e presentes na escola nas datas em que foram agendadas as visitas para a coleta de dados.

Os estudos de Lajeado e Sapiranga foram submetidos e aprovados pelo Comitê de Ética e Pesquisa da Universidade do Vale do Rio dos Sinos (Unisinos), conforme pareceres de no 074/2011 (Lajeado) e no 028/2012 (Sapiranga). Os gestores de rede (estadual ou municipal) e as direções das escolas particulares assinaram Termo de Anuência, indicando sua concordância em participar dos estudos, e os familiares dos escolares receberam e assinaram o Termo de Consentimento Livre e Esclarecido que Ihes foi apresentado. Os escolares sem autorização dos responsáveis para participar ou que não desejassem responder foram instruídos a receber o questionário e a devolvê-lo em branco, evitando-se, assim, a identificação dos não respondentes em sala de aula.

Foi realizado controle de qualidade por meio de acompanhamento de uma supervisora durante a coleta e checagem das visitas com as escolas, além da dupla entrada de dados no programa Epidata 3.1, e análise descritiva prévia, possibilitando a checagem de erros e inconsistências. Foram computadas, ao todo, entre recusas e exclusões por inconsistências, perdas da ordem de 9,4\% das entrevistas realizadas.

As análises tiveram como desfecho a referência ao uso no ano de álcool, tabaco, maconha ou cocaína, tomadas de modo independente. A exposição foi o relato manter atividade profissional pela qual recebesse remuneração. Tanto a exposição quanto o desfecho eram variáveis categóricas dicotômicas, do tipo "sim/não", como respostas obtidas a perguntas diretas.
O cálculo amostral considerou prevalências do desfecho entre os não expostos, variando de 1,1\% para cocaína a 40,2\% para álcool, com razão de não expostos para expostos de 1,42, razões de prevalência variando de 1,5 a 2,9, com nível de confiança de $95 \%$ e poder estatístico de $80 \%$, indicando o número de 1.680 entrevistas como a maior amostra necessária para as análises propostas. O grupo de escolares com 14 anos ou mais nas entrevistas realizadas totalizou 1.999 escolares. Desses, 38 (1,9\%) foram excluídos por falta de informação quanto à situação de trabaIho. O grupo de escolares que não responderam quanto à inserção no mercado de trabalho tinha comportamentos semelhantes aos demais quanto às variáveis em análise. A amostra final deste estudo foi composta por 1.961 escolares, com média de idade de 15,24 anos (desvio-padrão de 1,06).

As análises foram rodadas nos programas SPSS 20.0 e Stata 11.0, disponíveis na instituição sede do estudo e todas as etapas foram submetidas a controle para efeito de delineamento pela variável escola. Para as análises ajustadas das razões de prevalência, foram pensados dois modelos. O primeiro incluiu variáveis de características individuais dos escolares: idade e sexo. Idade em anos completos é variável discreta obtida pelo cálculo do tempo transcorrido entre ano e mês de nascimento e a data da coleta de dados, categorizada em: 14, 15, 16 ou 17 anos. Sexo é variável categórica dicotômica, autodeclarada como masculino ou feminino.

O segundo nível de análise considerou as variáveis anteriores mais a referência de supervisão dos cuidadores, indicado pela supervisão que os cuidadores, em casa, mantinham sobre o uso de internet, segundo o relato dos entrevistados. Essa é uma variável discreta, com as categorias: "não uso computador"; "uso e ninguém acompanha nem controla"; "uso e mostro o que faço de vez em quando"; "uso e alguém sempre acompanha o que faço".

\section{RESULTADOS}

A tabela 1 mostra a distribuição dos escolares segundo as variáveis de interesse nesta análise. Ter um trabalho onde recebe remuneração foi relatado por 41,3\% (IC 95\%: 39,3\%$43,2 \%)$ dos escolares, enquanto o uso no ano de tabaco foi referido por 13,9\% (IC 95\%: 12,4\%-15,4\%), álcool por 62,8\% (IC 95\%: 60,7\%-64,8\%), maconha por 7,8\% (IC 95\%: 6,7\%9,0\%) e cocaína por 2,8\% (IC 95\%: 2,0\%-3,5\%).

$\mathrm{Na}$ análise bruta por Regressão de Poisson, foi estimada associação significativa entre o trabalho remunerado e o uso no ano de tabaco [RP = 1,47 (IC 95\%: 1,19-1,84) $p<0,001]$, álcool [RP $=1,12$ (IC 95\%: 1,04-1,19) $p=0,001]$, maconha [RP $=1,35$ (IC 95\%: 1,00-1,82) $p=0,049]$ e cocaína $[R P=2,07$ (IC 95\%: 1,21-3,54) $p=0,008$ ] (Tabela 2). 
Tabela 1. Distribuição, em frequência relativa (\%), com respectivos intervalos de confiança (IC 95\%), dos escolares entre 14 e 17 anos de idade de duas cidades de porte médio do sul do Brasil, segundo as variáveis em análise - $2012(n=1.961)^{*}$

\begin{tabular}{|c|c|c|}
\hline Variáveis & $\%$ & IC 95\% \\
\hline \multicolumn{3}{|l|}{ Idade } \\
\hline 14 anos & 32,1 & $30,4 \%-33,8 \%$ \\
\hline 15 anos & 27,1 & $25,1 \%-29,0 \%$ \\
\hline 16 anos & 25,4 & $23,6 \%-27,2 \%$ \\
\hline 17 anos & 15,4 & $14,0 \%-17,0 \%$ \\
\hline \multicolumn{3}{|l|}{ Sexo } \\
\hline Masculino & 46,0 & $43,8 \%-48,1 \%$ \\
\hline Feminino & 54,0 & $51,9 \%-56,2 \%$ \\
\hline \multicolumn{3}{|c|}{ Supervisão parental no computador } \\
\hline Não usa & 14,3 & $12,8 \%-15,8 \%$ \\
\hline Sem supervisão & 44,2 & $42,0 \%-46,3 \%$ \\
\hline Supervisão eventual & 33,3 & $31,3 \%-35,4 \%$ \\
\hline Supervisão regular & 8,2 & $7,0 \%-9,4 \%$ \\
\hline \multicolumn{3}{|l|}{ Ter trabalho remunerado } \\
\hline Não & 58,7 & $56,8 \%-60,7 \%$ \\
\hline Sim & 41,3 & $39,3 \%-43,2 \%$ \\
\hline \multicolumn{3}{|l|}{ Uso de tabaco no ano } \\
\hline Não & 86,1 & $84,6 \%-87,6 \%$ \\
\hline Sim & 13,9 & $12,4 \%-15,4 \%$ \\
\hline \multicolumn{3}{|l|}{ Uso de álcool no ano } \\
\hline Não & 37,2 & $35,2 \%-39,3 \%$ \\
\hline Sim & 62,8 & $60,7 \%-64,8 \%$ \\
\hline \multicolumn{3}{|l|}{ Uso de maconha no ano } \\
\hline Não & 92,2 & $91,0 \%-93,3 \%$ \\
\hline Sim & $7,8 \%$ & $6,7 \%-9,0 \%$ \\
\hline \multicolumn{3}{|l|}{ Uso de cocaína no ano } \\
\hline Não & 97,2 & $96,5 \%-97,9 \%$ \\
\hline Sim & 2,8 & $2,0 \%-3,5 \%$ \\
\hline
\end{tabular}

Para a análise ajustada, foram adicionadas, num primeiro momento, as variáveis sexo e idade. Apenas para uso no ano de tabaco se manteve associação com o trabalho remunerado $[\mathrm{RP}=1,30$ (IC 95\%: 1,04-1,64) $\mathrm{p}=0,024)](\mathrm{Ta}-$ bela 3).

A relação entre o uso no ano de tabaco e a inserção no mercado de trabalho foi, então, submetida à análise ajustada para sexo, idade e também para o relato de supervisão no domicílio para o uso da internet. Não persistiu associação significativa nessa análise $[R P=1,23($ IC 95\% = 0,98-1,55) $p=0,079]$.

\section{DISCUSSÃO}

A prevalência estimada de inserção no mercado de trabalho de 41,3\% dos escolares com idades entre 14 e 17 anos, desses dois municípios de médio porte, com até 70 mil habitantes, no interior do Rio Grande do Sul, parece elevada se comparada ao que tem sido descrito em outros estudos, 10,13,16. As estimativas de uso no ano das substâncias estudadas, porém, acompanham o que tem sido descrito para a população dessa faixa etária 6 .

A associação entre atividade remunerada e uso de drogas, verificada aqui apenas na análise bruta, corrobora achados de outros autores que, inclusive, formulam algumas hipóteses para tal achado. Maior disponibilidade de dinheiro apresentou associação significativa com o uso de drogas ${ }^{12,16}$ e foi relatada em estudo qualitativo como razão para o consumo de substâncias psicoativas ${ }^{15}$. O contato com colegas que podem facilitar o consumo, tanto por serem mais veIhos quanto pelos ritos que podem envolver o trabalho, por exemplo, beber no final do expediente, também foi apontado como fator associado ao aumento do risco de uso de substâncias entre adolescentes ${ }^{14-16}$. Por se tratar de estudo

Tabela 2. Razões de prevalência (RP) brutas e respectivos intervalos de confiança de 95\% (IC 95\%) para uso no ano de substâncias segundo inserção no mercado de trabalho de escolares de 14 a 17 anos de idade, no sul do Brasil $-2012(n=1961)^{*}$

\begin{tabular}{|c|c|c|c|c|c|c|c|c|c|c|c|c|}
\hline \multirow{2}{*}{ Variável } & \multicolumn{3}{|c|}{ Tabaco } & \multicolumn{3}{|c|}{ Álcool } & \multicolumn{3}{|c|}{ Maconha } & \multicolumn{3}{|c|}{ Cocaína } \\
\hline & $\mathrm{RP}$ & IC95\% & $p$ & RP & IC $95 \%$ & $p$ & $\mathrm{RP}$ & IC $95 \%$ & $p$ & RP & IC $95 \%$ & $p$ \\
\hline Trabalha & & & $<0,001$ & & & 0,001 & & & 0,049 & & & 0,008 \\
\hline Não & 1 & & & 1 & & & 1 & & & 1 & & \\
\hline Sim & 1,47 & $1,19-1,84$ & & 1,12 & $1,04-1,19$ & & 1,35 & $1,00-1,82$ & & 2,07 & $1,21-3,54$ & \\
\hline
\end{tabular}

* Análises precedidas de controle para efeito de delineamento para variável escola.

Tabela 3. Razões de prevalência (RP) ajustadas para sexo e idade e respectivos intervalos de confiança de 95\% (IC 95\%) para uso no ano de substâncias segundo a inserção de escolares de 14 a 17 anos de idade no mercado de trabalho, no sul do Brasil - 2012 ( $n=1961)^{*}$

\begin{tabular}{|c|c|c|c|c|c|c|c|c|c|c|c|c|}
\hline \multirow{2}{*}{ Variável } & \multicolumn{3}{|c|}{ Tabaco } & \multicolumn{3}{|c|}{ Álcool } & \multicolumn{3}{|c|}{ Maconha } & \multicolumn{3}{|c|}{ Cocaína } \\
\hline & RP & IC $95 \%$ & $p$ & RP & IC $95 \%$ & $p$ & RP & IC $95 \%$ & $p$ & $\mathrm{RP}$ & IC $95 \%$ & $p$ \\
\hline Trabalha & & & 0,024 & & & 0,183 & & & 0,796 & & & 0,089 \\
\hline Não & 1 & & & 1 & & & 1 & & & 1 & & \\
\hline Sim & 1,30 & $1,04-1,64$ & & 1,05 & $0,98-1,12$ & & 1,04 & $0,76-1,43$ & & 1,64 & $0,93-2,89$ & \\
\hline
\end{tabular}


transversal, não é possível estimar causalidade, portanto, não se pode dizer que a inserção no mercado de trabalho levou ao consumo de drogas, já que também é possível que os escolares tenham buscado o trabalho remunerado como forma de obter o dinheiro necessário para o consumo, como sugere Do e Finkelstein ${ }^{13}$. Também não é possível, com esse delineamento, estimar que a inserção mais precoce no mercado de trabalho tenha efeito protetor ou preditor do comportamento uso de substâncias mais tarde, na idade adulta, por exemplo.

Outros estudos, porém, levam em conta a carga de trabalho do adolescente, concluindo que quanto maior a carga horária de trabalho, maior a associação com o uso de drogas $^{13,14}$. Longos períodos de trabalho também são associados com baixo desempenho acadêmico, estresse e outros problemas de saúde ${ }^{16}$, fatores que também podem ter relação com o abuso de substâncias. Ramchand et al. ${ }^{14}$ concluíram que, enquanto adolescentes com mais de 10 horas de trabalho por semana apresentaram risco aumentado de uso de tabaco, aqueles que trabalhavam até 10 horas na semana apresentaram menores riscos, mesmo quando comparados aos que não trabalhavam. Além disso, estudantes com carga de trabalho reduzida também apresentaram melhor desempenho escolar, maior comprometimento com a educação e maiores expectativas em relação a frequentar a universida$\mathrm{de}^{14}$. Esses resultados sugerem que a inserção no mercado de trabalho, quando observados a carga horária e o risco da atividade, pode ser fator protetor para o uso de substâncias e colaborar na vida escolar dos adolescentes. Neste estudo, a única informação acessada foi ter ou não um trabalho remunerado. Considera-se uma limitação, portanto, não ser possível ajustar as análises também para a influência da carga e do tipo de trabalho para essa amostra. Estudos futuros poderão ampliar o exame dessa questão, considerando ainda outras variáveis relacionadas ao trabalho, como o tipo de ocupação, as condições do ambiente de trabalho ou o perfil da organização. No trabalho em empresas familiares, por exemplo, tanto a supervisão, referida como protetora, quanto os conflitos do ambiente doméstico, percebidos como predisponentes para o consumo de substâncias, podem estar presentes.

Além disso, a amostra deste estudo é composta por adolescentes que mantêm o vínculo com a escola. A literatura cita prevalências ainda mais altas de uso de drogas entre trabalhadores que abandonaram a escola ${ }^{10,16}$. Dall'Agnol et al. ${ }^{10}$, em estudo realizado em Pelotas, também no interior do $\mathrm{RS}$, estimaram uma prevalência de uso de tabaco 6,3\% maior entre adolescentes que haviam deixado a escola. Nos Estados Unidos, Wu et al. ${ }^{16}$ observaram prevalências mais altas de consumo de álcool, tabaco e drogas ilícitas para adolescentes que estavam fora da escola. Esses resultados corroboram os estudos de Gallo e Williams ${ }^{19}$, que sugerem que a escola é um fator de proteção contra diversos comportamentos de risco, entre eles o uso de drogas. A evasão escolar é um fenômeno multifatoria ${ }^{20}$, no qual se inserem, entre outros, o uso de substâncias e a inserção no mercado de trabalho. Bray et al. ${ }^{21}$ realizaram estudo longitudinal examinando a relação entre o uso de maconha e a evasão escolar e concluíram que o início do uso de qualquer uma das substâncias estudadas aumentou o risco de evasão escolar. A inserção no mercado de trabalho não teve associação significativa com a evasão escolar no estudo de Entwisle et al.22 após a análise ajustada.

A supressão de significância estatística para a associação entre a exposição e os desfechos deste estudo, quando as análises são ajustadas para sexo e idade (o que ocorre para álcool, maconha e cocaína) e para sexo, idade e supervisão parental (o que ocorre para tabaco), indica que o consumo dessas substâncias tende a ocorrer progressivamente mais, assim como a inserção no mercado de trabalho, à medida que a idade avança, com perfis específicos, que variam segundo o sexo dos indivíduos e de acordo com características do funcionamento das relações familiares.

Um estudo realizado em uma cidade da Bahia ${ }^{23}$, envolvendo escolares de 14 a 19 anos, identificou risco aumentado de uso de álcool para adolescentes do sexo masculino, entre 17 e 19 anos, corroborando os resultados obtidos neste estudo. Outros estudos também verificaram que o aumento da idade e ser do sexo masculino aumentaram os riscos de consumo de drogas ${ }^{24,25}$.

Em relação às estratégias de cuidado implementadas pelos pais, Montgomery et al. ${ }^{26}$ obtiveram relato de maior controle exercido pelos pais entre adolescentes que não eram usuários de drogas, além de maior risco de uso de drogas na vida entre os adolescentes que tinham pais negligentes ou permissivos. Stephenson et al. ${ }^{27}$ citam controle e conhecimento acerca da rotina do filho como fatores protetores ao uso de drogas entre adolescentes.

Na literatura revisada acerca da relação entre trabalho e uso de drogas, alguns estudos controlaram suas análises para idade, sexo ${ }^{13}$ e monitoramento parental ${ }^{14}$, indicando que a relação entre essas variáveis, tanto com a inserção no mercado de trabalho quanto com o uso de substâncias, já foi observada. Além disso, Wu et al. ${ }^{16}$, ao analisarem o impacto da carga de trabalho no uso de substâncias, observaram que adolescentes mais velhos trabalhavam por períodos mais longos e também tinham risco aumentado de consumo. Godley et al. ${ }^{15}$, em estudo qualitativo com usuários em tratamento, destacaram que os adolescentes raramente relatavam o monitoramento dos pais quanto ao uso do dinheiro obtido no trabalho.

\section{CONCLUSÕES}

Mesmo sem dados que permitissem ajustar as análises para carga e tipo de trabalho, este estudo mostra que, num universo de adolescentes que mantinham vínculo com a escola, 
em dois municípios de médio porte do interior do país, onde a busca dos escolares por atividade profissional remunerada é elevada, essa condição não persiste associada ao uso no ano de tabaco, bebidas alcoólicas, maconha ou cocaína, em análise ajustada para sexo, idade e supervisão parental. Destacam-se a aproximação dos jovens a todos os mercados, do trabalho e das drogas, com o avanço da idade, bem como a importância da preservação do vínculo escolar e da supervisão parental.

\section{CONTRIBUIÇÕES INDIVIDUAIS}

Simone Poletto - Fez a revisão da literatura, participou da organização do banco de dados e coordenou a análise dos dados e a redação da versão final deste artigo.

Rogério Lessa Horta - Participou da elaboração dos projetos de base, coordenou a coleta dos dados, colaborou no plano de análise, na revisão da literatura, na análise dos dados e na redação da versão final do artigo.

Vanessa Andina Teixeira - Elaborou plano de análise, colaborou na revisão de literatura e aprovou a versão final do artigo.

Valenca Lemes Grapiglia e Alexandre Dido Balbinot - Colaboraram na organização do banco de dados e aprovaram a versão final do artigo.

\section{CONFLITOS DE INTERESSE}

Os autores declaram não haver conflitos de interesse.

\section{AGRADECIMENTOS}

Estudo financiado com recursos do CNPq: Edital Universal 2013, processo 470691/2013-1.

\section{REFERÊNCIAS}

1. Cavalcante MBPT, Alves MDS, Barroso MGT. Adolescência, álcool e drogas: uma revisão na perspectiva da promoção da saúde. Esc Anna Nery Rev Enferm. 2008;12(3):555-9.

2. Matos AM, Carvalho RC, Costa MCO, Gomes KEPS, Santos LM. Consumo frequente de bebidas alcoólicas por adolescentes escolares: estudo de fatores associados. Rev Bras Epidemiol. 2010;13(2):302-13.

3. Almeida Filho JA, Ferreira MA, Gomes MLB, Silva RC, Santos TCF. 0 adolescente e as drogas: consequências para a saúde. Esc Anna Nery Rev Enferm. 2007;11(4):606-10.

4. Soldera M, Dalgalarrondo P, Corrêa Filho HR, Silva CAM. Uso de drogas psicotrópicas por estudantes: prevalência e fatores sociais associados. Rev Saúde Pública. 2004;38(2):277-83.
5. Pinsky I, El Jundi SARJ. 0 impacto da publicidade de bebidas alcoólicas sobre 0 consumo entre jovens: revisão da literatura internacional. Rev Bras Psiquiatr. 2008;30(4):362-74.

6. Carlini ELA, Noto AR, Sanchez ZM, Carlini CMA, Locatelli DP, Abeidet LR, et al. VI Levantamento Nacional sobre o Consumo de Drogas Psicotrópicas entre Estudantes do Ensino Fundamental e Médio das Redes Pública e Privada de Ensino nas 27 Capitais Brasileiras - 2010. Centro Brasileiro de Informações sobre Drogas Psicotrópicas (Cebrid); Universidade Federal de São Paulo (Unifesp); Secretaria Nacional de Políticas sobre Drogas (Senad); Ministério da Justiça; Governo Federal. 2010.

7. Pratta EMM, Santos MA. Lazer e uso de substâncias psicoativas na adolescência: possíveis relações. Psic Teor Pesq. 2007;32(1):43-52.

8. Schenker M, Minayo MCS. Fatores de risco e de proteção para o uso de drogas na adolescência. Ciênc Saúde Coletiva. 2005;10(3):707-17.

9. Tavares BF, Béria JU, Lima MS. Fatores associados ao uso de drogas entre adolescentes escolares. Rev Saúde Pública. 2004;38(6):787-96.

10. Dall'Agnol MM, Fassa AG, Facchini LA. Child and adolescent labor and smoking: a crosssectional study in southern Brazil. Cad Saude Publica. 2011;27(1):46-56.

11. Silva VA, Aguiar AS, Felix F, Rebello GP, Andrade RC, Mattos HF. Brazilian study on substance misuse in adolescents: associated factors and adherence to treatment. Rev Bras Psiquiatr. 2003;25(3):133-8.

12. McCrystal P, Percy A, Higgins K. The cost of drug use in adolescence: young people, money and substance abuse. Drugs Educ Prev Polic. 2007;14(1):19-28.

13. Do YK, Finkelstein EA. Youth employment, income, and smoking initiation: results from Korean panel data. J Adolesc Health. 2012;51:226-32.

14. Ramchand R, lalongo NS, Chilcoat HD. The effect of working for pay on adolescent tobacco use. Am J Public Health. 2007;97(11):2056-62.

15. Godley SH, Passetti LL, White MK. Employment and adolescent al cohol and drug treatment and recovery: an exploratory study. Am J Addict. 2006;15 Suppl 1:137-43.

16. Wu LT, Schlenger WE, Galvin DM. The relationship between employment and substance use among students aged 12 to 17. J Adolesc Health. 2003;32(1):5-15.

17. Gonçalves H, Menezes AMB, Bacchieri G, Dilélio AS, Bocanegra CAD, Castilhos ED, et al. Perfil de trabalho urbano de adolescentes de 14-15 anos: um estudo populacional no sul do Brasil. Ciênc Saúde Coletiva. 2012;17(5):1267-74.

18. Brasil. Lei no 10.097 , de 19 de dezembro de 2000. Altera dispositivos da Consolidação das Leis do Trabalho - CLT, aprovada pelo Decreto-Lei no 5.452, de 10 de maio de 1943. Disponível em: <http://www.planalto.gov.br/ccivil_03/leis/110097.htm>. Acesso em: 16 mar. 2014.

19. Gallo AE, Williams LCA. A escola como fator de proteção à conduta infracional de adolescentes. Cad Pesqui. 2008:38(133):41-59.

20. Townsend L, Flisher AJ, King G. A systematic review of the relationship between high school dropout and substance use. Clin Child Fam Psychol Rev. 2007;10(4):295-317.

21. Bray JW, Zarkin GA, Ringwalt C, Qi J. The relationship between marijuana initiation and dropping out of high school. Health Econom. 2000;9(1):9-18.

22. Entwisle DR, Alexander KL, Olson LS. Urban teenagers: work and dropout. Youth Soc. 2005;37(1):3-32.

23. Matos AM, Carvalho RC, Costa MCO, Gomes KEPS, Santos LM. Consumo frequente de bebidas alcoólicas por adolescentes escolares: estudo de fatores associados. Rev Bras Epidemiol. 2010;13(2):302-13.

24. Baus J, Kupek E, Pires M. Prevalência e fatores de risco relacionados ao uso de drogas entre escolares. Rev Saúde Pública. 2002;36(1):40-6.

25. Jinez MLJ, Souza JRM, Pillon SC. Uso de drogas e fatores de risco entre estudantes de ensino médio. Rev Latino-am Enfermagem. 2009;17(2).

26. Montgomery C, Fisk JE, Craig L. The effects of perceived parenting style on the propensity for illicit drug use: the importance of parental warmth and control. Drug Alcohol Rev. 2008;27(6):640-9.

27. Stephenson MT, Quick BL, Atkinson J, Tschida DA. Authoritative parenting and drugprevention practices: implications for antidrug ads for parents. Health Commun. 2005;17(3):301-21. 